\begin{tabular}{|c|c|}
\hline Title & Spin-flip quenching in trion state mediated by optical phonons in a single quantum dot \\
\hline Author(s) & Kumano, H.; Ekuni, S.; Kobay ashi, H.; Sasakura, H.; Suemune, I.; A dachi, S.; Muto, S. \\
\hline Citation & $\begin{array}{l}\text { Phy sica Status Solidi B Basic Research, 246(4), 775-778 } \\
\text { https://doi.org/10.1002/pssb.200880619 }\end{array}$ \\
\hline Issue Date & 2009-04 \\
\hline DOC URL & http:/hdl. handle.net/2115/45359 \\
\hline Rights & $\begin{array}{l}\text { This is the pre peer reviewed version of the following article: phy sica status solidi (b)V olume 246, Issue 4, pages } \\
775-778 \text {, A pril 2009, which has been published in final form at } \\
\text { http://onlinelibrary.wiley.com/doi } / 0.1002 / p s s b .200880619 / \text { abstract. }\end{array}$ \\
\hline Type & article (author version) \\
\hline File Information & 286_suemune.pdf \\
\hline
\end{tabular}

Instructions for use 


\title{
Spin-flip quenching in trion state mediated by optical phonons in a single quantum dot
}

\author{
H. Kumano ${ }^{1,2}$, S. Ekuni ${ }^{1}$, H. Kobayashi ${ }^{1}$, H. Sasakura ${ }^{1}$, I. Suemune ${ }^{1,2}$ \\ S. Adachi ${ }^{3}$, and S. Muto ${ }^{3}$ \\ ${ }^{1}$ Research Institute for Electronic Science, Hokkaido University, Sapporo, Japan \\ ${ }^{2}$ CREST, Japan Science and Technology Agency, Kawaguchi, Japan \\ ${ }^{3}$ Applied Physics, Hokkaido University, Sapporo, Japan
}

Received $z z z$, revised $z z z$, accepted $z z z$

Published online $z z z$

PACS 71.35.-y, 72.25.Fe, 72.25.Rb, 78.67.Hc

High degree of preservation of spin states mediated by optical phonons is demonstrated with a positive trion state in a single quantum dot. At the optical phonon resonance, suppression of spin relaxation is clearly identified as dip structures in photoluminescence excitation spectra under cross-circularly polarized detection with respect to a pumping. As a result, distinguishably high degree of circular polarization up to $\sim 0.85$ is achieved in the absence of external magnetic field, which is not the case for neutral excitons with finite fine structure splitting. The absence of continuum states plays a crucial role for this observation.

\section{Introduction}

With respect to strong demand for secure communication network, quantum key distribution $(\mathrm{QKD})$ is attracting much more attention due to its highly secure nature [1]. Since it is based on physical principles such as indivisibility and non-cloning of single photons instead of mathematical complexity, completely secure communication which is free from eavesdropping will be possible in principle. One of the most important protocols for implementing the QKD is BB84 [2]. Since the BB84 is based on photon polarization states belonging to two non-orthogonal bases, generation of single photons from quantum dot (QD) with deterministic polarization state is prerequisite for coding. When the QD is taken as an on-demand "photon-state converter" from coherent state of excitation laser to single photon train in number state for the BB84, polarization fluctuation between emitting photons after each excitation events will strongly reduce the consequently available bit rate of quantum-optical devices since the specific photon polarization is filtered for the subsequent polarization modulation. Therefore, efficient polarization conversion between electron spin in a QD and emitted single photon is required, then the suppression of spin flip during whole processes including absorption, capture, relaxation, and radiative recombination is a crucial issue. In case of neutral exciton, long-range electron-hole exchange interaction stems from an anisotropic confinement potential [3-5] will lead to a simultaneous spin flip of electron and hole [6] and also to lift the exciton state degeneracy. Singly charged exciton (trion), on the other hand, is free from the exchange interaction due to the presence of spin-paired carriers. Hence the spin flip between trion ground states will be well suppressed during its lifetime. Furthermore, since the trion is a half spin system, its ground states are degenerated due to Kramer's theorem in the absence of a magnetic field $[7,8]$, and the trion couples to circular-polarized photons guaranteed by a selection rule $[9,10]$. Therefore, the trion in a QD is quite promising for the efficient photon state converter, where its spin state formed by the circularly-polarized excitation in coherent state will be transferred into photons in number state with the incident polarization being preserved.

In this paper, single photon emission from a positive trion with high degree of circular polarization (DCP) is demonstrated without magnetic field. At the opticalphonon resonant excitation, suppression of spin relaxation is clearly identified as dip structures in photoluminescence excitation spectra detected by a cross-circular polarization. Resultant DCP amounts to $\sim 0.85$, which indicates that mutual polarization conversion from incident photon into sin- 
gle electron spin, and subsequent re-conversion into photon polarization in number state is realized with high efficiency.

\section{Experimental}

The $\mathrm{In}_{0.75} \mathrm{Al}_{0.25} \mathrm{As}$ QDs sample was grown on a semiinsulating (001)-GaAs substrate by molecular-beam epitaxy. The QDs were prepared in Stranski-Krastanow (S-K) growth mode on $\mathrm{Al}_{0.3} \mathrm{Ga}_{0.7} \mathrm{As}$ layers and were sandwiched with $\mathrm{Al}_{0.3} \mathrm{Ga}_{0.7} \mathrm{As}$ layers. The topmost surface was terminated with a GaAs cap layer. After the growth, the sample was etched into mesa structures with diameters of $\sim 150 \mathrm{~nm}$ for isolating single QD from the dot ensemble with the density of around $5 \times 10^{10} \mathrm{dots} / \mathrm{cm}^{2}$.

In this work, two QDs ( $Q D 1$ and $Q D 2)$ were investigated. A continuous-wave Ti: sapphire laser was used as an excitation source. An objective lens with the numerical aperture (NA) of 0.42 focused the laser beam on one of the mesa structures and collected luminescence emitted from the mesa. Collected luminescence was dispersed by a 0.64-m triple monochromator and introduced to a liquidnitrogen cooled Si charge-coupled-device detector. The PL polarization was analyzed in rectilinear $(\mathrm{H}, \mathrm{V})$ and/or circular $\left(\sigma^{+}, \sigma^{-}\right)$bases by a set of half/quarter-wave plate and a fixed Glan-Thomson linear polarizer in front of the monochromator, where $\sigma^{+}\left(\sigma^{-}\right)$denotes the circular polarization with a helicity of $+1(-1)$. The overall system resolution was $4.5 \mu \mathrm{eV}$ and all the measurements were done at $22 \mathrm{~K}$.

\section{Results and discussion}

PL spectrum from a $Q D 1$ is shown in Fig. 1(a). In this $\mathrm{QD}$, neutral exciton $\left(\mathrm{X}^{0}\right)$ and neutral biexciton $\left(\mathrm{XX}^{0}\right)$ were dominant, which were assigned and confirmed by excita-
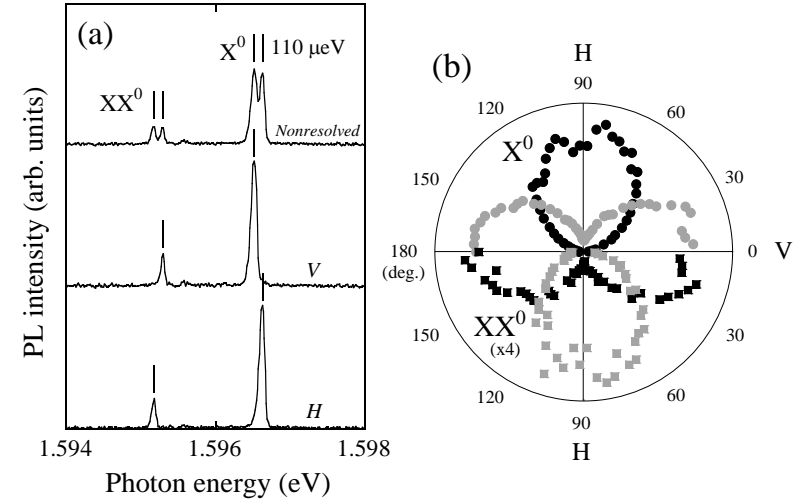

Figure 1 (a) PL spectra from a $Q D 1$ measured by nonpolarization resolved, vertical, and horizontal polarized detections. Fine structure splittings were clearly resolved for both $\mathrm{X}^{0}$ and $\mathrm{XX}^{0}$. (b) Detection polarization angle dependence of PL intensities of the $\mathrm{X}^{0}$ and $\mathrm{XX}^{0}$ emissions. Black circles (squares) are PL intenisty of high energy component of the $\mathrm{X}^{0}\left(\mathrm{XX}^{0}\right)$ emissions and gray circles (squares) are that of low energy component of the $\mathrm{X}^{0}\left(\mathrm{XX}^{0}\right)$ emissions. tion power dependence and lifetime measurements [11]. When PL was measured without selecting polarization, both $\mathrm{X}^{0}$ and $\mathrm{XX}^{0}$ had a doublet structure with identical energy splitting of $110 \mu \mathrm{eV}$. By selecting a rectilinear basis, each emission lines were exclusively detected when the detection polarization matched to the in-plane crystalline axes as shown in Fig. 1 (a). Furthermore, as shown in Fig. 1(b), clear anti-correlated angle dependence between $\mathrm{X}^{0}$ and $\mathrm{XX}^{0}$ was observed, which exhibits the fine structure splitting (FSS) in neutral exciton states [12]. This resulted in hindering single photon emission with circular polarization, and this was also the case for $Q D 2$ as shown in the inset of Fig. 4. On the other hand, in the case of QD2, charged exciton (positive trion) $\mathrm{X}^{+}$could be selectively populated in a QD by intra-dot excitation [11] and the PL spectrum is displayed in Fig. 2(a).

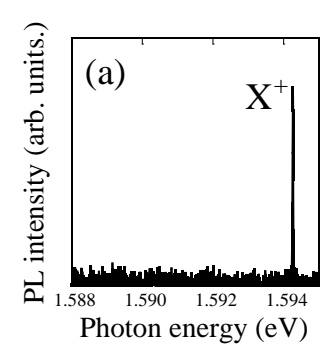

Photon energy (eV)

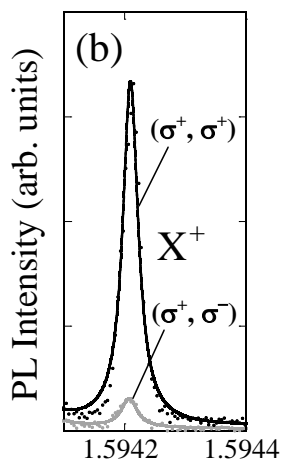

Photon Energy (eV)
Figure 2 (a) Single $\mathrm{X}^{+}$smission from a $Q D 2$ with an intra-dot excitation. (b) Polarization-dependent PL spectra under InAs LO phonon-resonant excitation. DCP amounts to $\sim 0.85$ for the $\mathrm{X}^{+}$emission without external magnetic field.

As expected, the $\mathrm{X}^{+}$emission showed no FSS, which indicates that two positive trion states with their total spin of $\pm 1 / 2$ are degenerated due to the absence of $e$ - $h$ exchange interaction. This feature is quite favorable for preserving spin states. Figure 3 shows the polarization-selective PLE spectra measured with circular basis under $\sigma^{+}$excitation as a function of the excitation energy relative to the detected $\mathrm{X}^{+}$emission. Here the energy resolution of the excitation energy was about $50 \mu \mathrm{eV}$. The PLE spectrum measured with the $\sigma^{-}$detection (hereafter designated as $\sigma^{-}$-PLE) was multiplied by 4.7 to clearly show the spectral difference to the $\sigma^{+}$detection. The DCP defined by $\left(I^{+}-\Gamma\right) /\left(I^{+}+\Gamma\right)$, where $I^{+}(I)$ denotes the PLE intensity measured in the $\sigma^{+}$ $\left(\sigma^{-}\right)$polarization, is also shown in Fig. 3. The multiplication factor of 4.7 given above for the $\sigma^{-}$-PLE corresponds to the average DCP of 0.65 measured under the non-resonant condition. Two peaks observed in the DCP almost agree with the energies of InAs LO (30.5 $\mathrm{meV}$ ) and TO (27.4 meV) phonon resonances [13]. The comparison of the PLE spectra with the co-circular and 


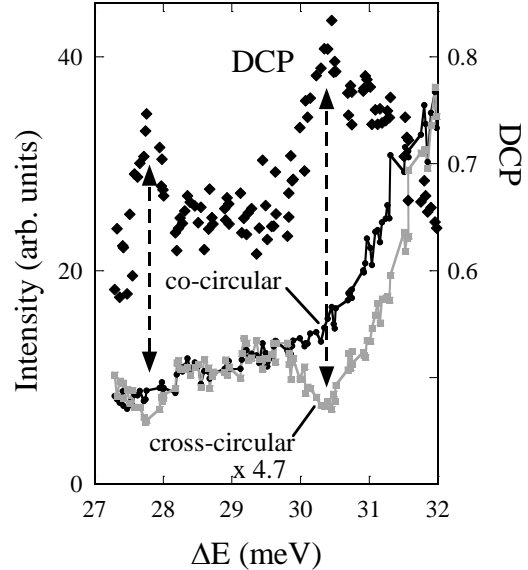

Figure 3 Polarization-selective PLE spectra of $Q D 2$ under $\sigma^{+}$excitation. Excitation energy is measured from the $\mathrm{X}^{+}$emission energy. The PLE spectrum with crosscircular detection to the pump is multiplied by 4.7. Peak structures in DCP and corresponding dip structures in PLE indicated by arrows are attributed to InAs LO (30.5 meV) and TO $(27.4 \mathrm{meV})$ phonon resonances.

cross-circular polarized detections show that these DCP peaks originate from the reduction of the cross-polarized $\sigma^{-}$ detection near the LO and TO resonances.

In general, the polarization-selective PLE spectra involve spin flips experienced both during and after energy relaxation processes to the $\mathrm{X}^{+}$state. The latter, i.e., spin flips between the two spin-degenerate $\mathrm{X}^{+}$ground states, is essentially considered to be independent of the excitation energy, hence the excitation-energy dependence of the polarization-selective PLE spectra predominantly reflects the spin-flips during the energy relaxation. Thus, the dip structures at the LO and TO phonon energies observed in the $\sigma^{-}$-PLE spectrum are the direct evidence of the suppressed spin-flips during the energy relaxation mediated by the optical phonons.

As a consecuqnce of the efficient quenching of spin flip during the energy relaxation, distinguishably high DCP is achieved for the $\mathrm{X}^{+}$emission. Figure 2(b) exhibits striking difference between PL intensities detected with co-circular and cross-circular configuration under the InAs LO phonon resonant excitation. In this condition, the DCP is as high as 0.85 without external magnetic field. This indicates that the spin polarization is highly stabilized by the InAs LO phonon resonant excitation. It is worth mentioning that the observation of the dip structure in the $\sigma^{-}$-PLE was largely owes to the critical spectral separation from the continuum background transitions [14]. Actually, continuum tail states were found to be extended from the WL edge down to $\sim 35 \mathrm{meV}$ above the e mission line in wide-range PLE spectrum under linear polarized excitation/detection condition (see Fig. 4). The increase of the PLE intensities observed above $\sim 31 \mathrm{meV}$ in Fig. 3

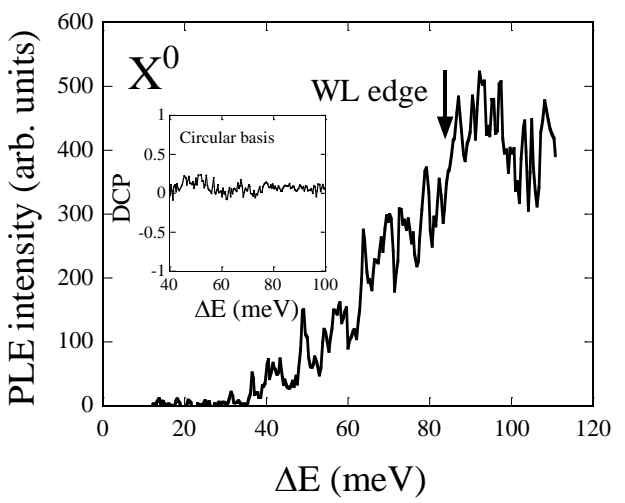

Figure 4 PLE spectra detected by $\mathrm{X}^{0}$ under linearpolarized excitation. Below the WL edge, tailed continuum states extend down to $\sim 35 \mathrm{meV}$ above the emission energy. (inset) Excitation energy dependence of the degree of circular polarization for $\mathrm{X}^{0}$. In contrast to charged trion, photon emission with circular polarization no longer occurred due to the finite FSS in the $\mathrm{X}^{0}$ states.

is then attributed to the onset of the continuum background transitions.

\section{Conclusions}

We have demonstrated highly efficient preservation of spin states mediated by optical phonons with a trion state in a single quantum dot. At the optical-phonon resonance, distinguishably high degree of circular polarization up to $\sim 0.85$ is achieved with a positive trion state in the absence of external magnetic field. This is not the case for neutral excitons with finite fine structure splitting. This indicates that the spin polarization is well stabilized by the InAs LO phonon resonant excitation.

\section{Acknowledgements}

The authors would like to acknowledge Dr. H. Z. Song, S. Hirose and M. Takatsu for the sample preparation. The authors are also grateful to Dr. M. Endo for his technical assistance. This work was supported in part by the Grant-in-Aid for Scientific Research (S)(2), No. 16106005, Young Scientists (A), No. 18681025, and Hokkaido Innovation Through Nanotechnology Supports (HINTs) from the Ministry of Education, Culture, Sports, Science and Technology.

\section{References}

[1] N. Gisin, G. Ribordy, W. Tittel, and H. Zbinden, Rev. Mod. Phys. 74, 145 (2002).

[2] C. H. Bennett and G. Brassard, Proc. of IEEE Int. Conf. on Comp. Sys. and Signal Proc., Bangalore, India 175 (1984).

[3] C. Santori, D. Fattal, M. Pelton, G. S. Solomon, and Y. Yamamoto, Phys. Rev. B 66, 045308 (2002).

[4] R. J. Young, R. M. Stevenson, A. J. Shields, P. Atkinson, K. Cooper, D. A. Ritchie, K. M. Groom, A. I. Tartakovskii, and M. S. Skolnick, Phys. Rev. B 72, 113305 (2005).

[5] R. Seguin, A. Schliwa, T. D. Germann, S. Rodt, K. Pötschke, A. Strittmatter, U. W. Pohl, D. Bimberg, M. Winkelnkemper, T. 
Hammerschmidt, and P. Kratzer, Appl. Phys. Lett. 89, 263109 (2006).

[6] E. Tsitsishvili, R. V. Baltz, and H. Kalt, Phys. Rev. B 67, 205330 (2003).

[7] M. Bayer, G. Ortner, O. Stern, A. Kuther, A. A. Gorbunov, and A. Forchel, P. Hawrylak, S. Fafard, and K. Hinzer, T. L. Reinecke and S. N. Walck, J. P. Reithmaier, F. Klopf, and F. Schäfer, Phys. Rev. B 65, 195315 (2002).

[8] I. A. Akimov, A. Hundt, T. Flissikowski, and F. Henneberger, Appl. Phys. Lett., 81, 4730 (2002).

[9] M. E. Ware, A. S. Bracker, E. Stinaff, D. Gammon, D. Gershoni and V. L. Korenev, Physica E 26, 55 (2005).

[10] T. Flissikowski, I. A. Akimov, A. Hundt, and F. Henneberger, Phys. Rev. B 68, 161309(R) (2003).

[11] H. Kumano, S. Kimura, M. Endo, H. Sasakura, S. Adachi, S. Muto, and I. Suemune, J. Nanoelectron. Optoelectron. 1, 39 (2006).

[12] V. D. Kulakovskii, G. Bacher, R. Weigand, T. Kümmell, A. Forchel, E. Borovitskaya, K. Leonardi, and D. Hommel. Phys. Rev. Lett., 82, 1780 (1999).

[13] D. L. Stierwalt and R. F. Potter, Phys. Rev. 137, 1007 (1965)

[14] Y. Toda, O. Moriwaki, M. Nishioka, and Y. Arakawa, Phys. Rev. Lett. 82, 4114 (1999). 